\title{
Validation Tool for Smart Screening of Language Disorders in Pediatrics Care
}

\author{
María Luisa Martín Ruiz, Miguel Angel Valero, and Iván Pau De Cruz
}

\begin{abstract}
Primary-care pediatricians could play a key role in early detection of development disorders as quick as they might have enough time and knowledge for suitable screenings at clinical routine. This research paper focuses on the development and validation of a knowledge-based web tool whose aim is to support a smart detection of developmental disorders in early childhood. Thus, the use of the system can trigger the necessary preventive and therapeutic actions from birth until the age of six. The platform was designed on the basis of an analysis of significant 21 cases of children with language disorders that supported the creation of a specific knowledge base, its ontology and a set of description logic relations. The resulting system is being validated in a scalable approach with a team of seven experts from the fields of neonathology, pediatrics, neurology and language therapy.
\end{abstract}

\section{Introduction}

Primary health care in Spain is currently close to collapse due to the high number of patients seen by a doctor through traditional consultations. In order to attend all the patients, they have seen reduced the time shared with the doctor in a consultation. This situation makes the quality of that care being eroded from the clinical point of view and a sense of lack of follow-up care received by the patients. Children, when are patients, are in the same situation.

This research focuses on promoting the welfare of children and their families. The early and appropriate diagnosis for the child's health status can lead to better treatment and effect on their development. The problems that could be detected in a routine visit to the pediatrician should be referred without delay to the appropriate specialist. This way the specialist can identify the disorder in the first stages and provide a proper medical treatment. This work aims to facilitate the task of pediatricians in the diagnosis and monitoring of children with language disorders. We propose a Decision Support System (DSS) that complements the actions of the pediatricians in the state of saturation in which they find themselves, helping them to find out early signs of language disorders. Thus, it is intended to catalyze processes of early intervention, early 
diagnosis and therapy, to improve the care of children with possible developmental disorders.

Primary-care pediatricians use information systems that fulfill the WONCA standards. Spanish healthcare system uses OMI, which does not gather aspects of the children's neuro-evolutive development and only it is thought to register information. Primary-care pediatricians play a key role in early detection of development alterations as they can undertake the preventive and therapeutic actions necessary in the interest of a child's optimal development. Present rates of detection of development disorders are lower than their real incidence [1], which means that early identification of children with such disorders remains a pending task. For these reasons, it is considered to be particularly valuable for a pediatrician to have access to an intelligent healthcare system in primary care to enable efficient screening of such disorders. This research paper describes the process of building of a tool for smart screening of language disorders in pediatrics care.

\section{Background}

Medicine is one of the fields to have benefited most from the use of computers, as a pioneer in the use of Decision Support Systems (DSS). HEPAXPERT (1991, Austrian) embedded knowledge-based diagnostic intelligence to interpret hepatitis serology test results. VIE-PNN was an expert system used for parenteral nutrition of neonates, (1993, Austrian). ERA project (2001, UK) is a DDS for interactive decision-making for identifying patients suspected of having cancer. ATENÍA project (2002, US) was used to control of hypertension in primary care. LISA project (2004, UK) assists in decision-making for children with lymphoblastic leukemia and the SimulConsult tool (2008, US) can detect health problems in children. In our study of DDS in medicine, no tool was found for the early detection of language disorders in children. Early detection of development disorders is very important, however, in clinical practice, pediatricians do not have enough time to perform proper screening of the children's neuro-evolutionary development, and their background in disabilities is not always as complete as would be desirable [2].

The use of smart systems in primary care becomes of greater interest as it can allow for detection of neurological disorders in children and prevention of added pathologies, achievement of functional improvements and allow for a more adaptive adjustment between a child and his or her surroundings. Paul and Fejerman studies stressed that a complete neurological and pediatric evaluation can reveal related developmental disorders, starting with detection of a language disorder [3,4]. Narbona highlights the fact that a neurological disorder does not manifest itself solely in a delay in the correct acquisition of speech and language, although a delay in the correct acquisition of language is the first alarm sign of a future neurological disorder [5]. Although medical procedures are available to detect a number of neurological disorders in children [1], these procedures are difficult to apply in primary pediatric care, as many require significant time and specialized knowledge. The review in this research found no solutions that exploit the potential of DSS in combination with artificial intelligence to efficiently and effectively assist pediatricians in the early detection of these disorders. 


\section{Methodology}

The following team of experts from the field of healthcare participated in the construction of the Knowledge Base (KB) needed to enable knowledge inference in the DSS to perform early screening of language disorders in primary care: a neonatologist with high expertise in development disorders and child disability, former director of the neonatology department of San Carlos Hospital in Madrid, two primary care pediatricians, a neuropediatrician presently working in the Quirón Hospital of Madrid and two experts in specific language impairment who are therapists at the Language Intervention Center (LIC) at La Salle Campus (UAM) of Madrid.

The acquisition and systematization of needed knowledge required for the early referral of a DSS of a smart system proposed in this paper is a critical aspect that determines its effective use in primary care. The process of Knowledge Acquisition (KA) is the first step for creating a DSS and it strongly influences the conditions for correct operation. This process covers up to the final stage of DSS development. The methodology for KA requires consideration of both the definition of the knowledge to be systematized and the conceptualization and formal design of the information compiled from human and materials sources in order to model the functioning of the DSS [6]. The main methodologies available for extracting knowledge were studied (GROVER, CommonKADS (CK) [6], Methontology [7] and IDEAL). The conclusion of this study was to use a combination of $\mathrm{CK}$ and Methontology. The application of CK for the system design provided a set of early detection items to be considered by the pediatrician. This structured knowledge reflects all important aspects of the DSS to be implemented and verified through a user tool. CK was used in KA meetings as it is most suitable for modeling the knowledge extracted from language specialists in the form of ontology. Methontology defines a set of tasks that enabled moving from an informal specification of the domain of application, collected with the language specialists, to a semi-formal specification of the domain. This facility makes easier the understanding of the ontology for smart screening of language disorders by consulted neuropediatricians and language therapists as well as the system developer. Figure 1 summarizes the empirical design methodology for the construction of the DSS.

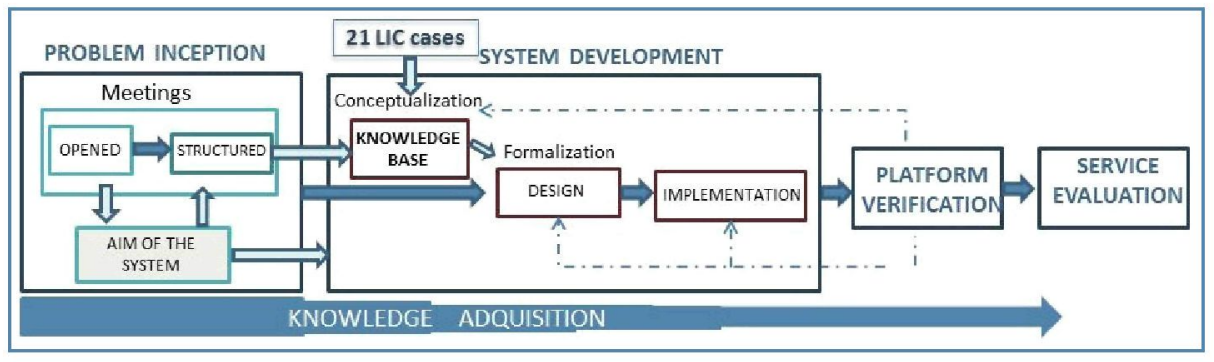

Fig. 1. Development methodology 
DSS development has been carried out in four steps:

Problem inception: aims to fully define the problem to be solved for early intervention. In this phased, the group of experts involved in the KA process were - a pediatrician, a neuropediatrician and a neonatologist- held five open meetings between September 2009 and May 2011 in order to fully define the problem to be solved, work on early screening of language disorders, with the construction of a smart detection system.

System development: includes the implementation phase of the DSS. The main result is the $\mathrm{KB}$. In this phased, the group of experts were - a neuropediatrician and two experts in specific language impairment who are therapists at the Language Intervention Center (LIC) at La Salle Campus (UAM) of Madrid specialized in neurological disorders in children - created the KB through ten structured meetings. The process of building and purging the $\mathrm{KB}$ was developed through a retrospective analysis of information on levels of language acquisition of 21 children who received therapy in the LIC [8]. The KB has been developed using Protégé (ontology creation platform) [9] and Pellet (reasoning engine) [10]. The verification of the KB required a usable tool so that specialists might interact with the KBS in an efficient way.

Platform verification: experts should be able to evaluate whether the system proposal to refer to a specialist arising from a detection of language development disorders was correct or not. Thus, a web tool (Figure 2) was built to facilitate the work of experts and primary care pediatricians. Five specialists have been involved in the verification stages both for usability and system performance tests along six months (two pediatricians, two language therapists and one neuropediatrician). This process contributed in a satisfactory way to improve the graphical user interface and the reasoning rules of the KB. End users pointed out that they could use the tool by themselves in clinical routine.

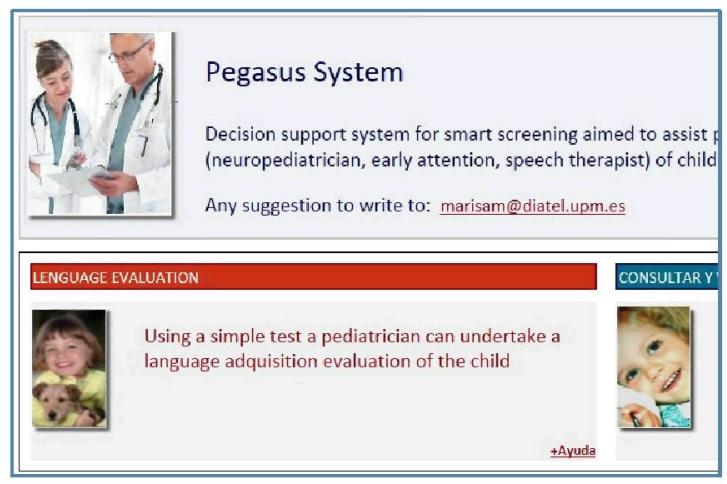

Fig. 2. Access to the early detection tool of language disorders

Service evaluation: This evaluation is scheduled for autumn 2012. We will evaluate the results by means of a control of false positives and negatives according to a sample give 100 children chosen at random by the pediatricians who will take part in the evaluation phase. 


\section{$4 \quad$ Results}

This section contains the main results of this research. First, it describes the general System Architecture. Secondly, it describes the construction of the KB and then the process of formalization of the KB. Lastly, an example is shown of an ad-hoc web interface for verification of the resulting $\mathrm{KB}$.

\subsection{General System Architecture}

The functional architecture of the resulting system must facilitate dynamic interaction between the actors involved, distributed platforms for the management of information, models of reasoning and processes in line with the health care model in which it is located (Figure 3).

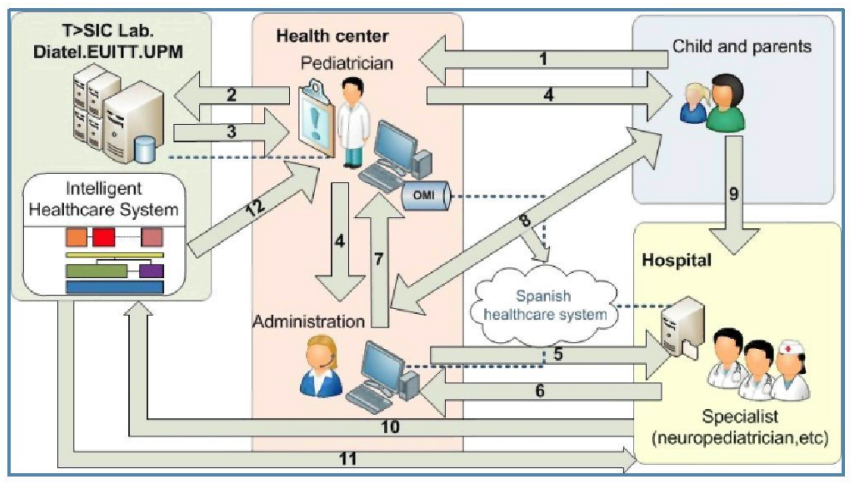

Fig. 3. General System Architecture

1. The child goes to the family pediatrician accompanied by a family member.

2. Primary health-care doctor decides to use the DSS to assess whether there is a language disorder in the child. The doctor will introduce the required information.

3. The DSS returns the result to the pediatrician.

4. Three possibilities:

(A) The result is that everything is normal.

(B) The result changes the visitation schedule the child's pediatrician, or the DSS proposed to be derived from the relevant specialist hospital. In this case the doctor decides whether to accept the decision of DSS and pursue the request for appointment at the hospital, or decide it is not necessary. As always the decision of DSS support to the pediatrician, never an imposition on the decisions that the doctor makes.

5. An appointment with the specialist is requested to the hospital

6. Response is received to the request for appointment with the hospital,

7. The details of the appointment with the specialist will be received by the pediatrician.

8. The appointment details reach the child and his family. 
9. The child goes to the specialist.

10. The specialist checks the response of the DSS for the corresponding case.

11. The DSS returns the result of the evaluation process for that case.

12. The DSS notifies the pediatrician that the specialist has accessed to the system.

The specialist will validate the outcome of DSS in order to improve the KB.

\subsection{Knowledge Base for Language Disorders}

The KB has been built through an iterative process of structured meetings between September 2011 and May 2012. Only the two language therapists and the neuropediatrician participated in the meetings, using $\mathrm{CK}$ techniques to extract information such as structured interviews to complete the $\mathrm{KB}$ with questions to be asked by the primary care pediatrician children's tutor upon arriving at the care facility. The questions were sharpened to focus on language, with a view to enabling the primary care pediatrician to detect possible delays in children's development that closer attention or immediate referral to an appropriate specialist. The process of building and purging the $\mathrm{KB}$ is based on the experience of LIC, who checked developmental items against the appearance of language disorders through a retrospective analysis of information on levels of language acquisition of 21 children who received therapy in the LIC [8].

The structuring of the final KB consists of 136 questions between month 1 and month 72 in the life of the child, and questions may be of two types:

- Questions called Alert Milestones that imply bringing forward the visit. The child makes a return visit to allow for re-evaluation of the level of language acquisition.

- Questions called Alarm Milestones that imply referral because they are considered reasons for alarm.

Table 1 details the KB questions for a child between the ages of 1 and 4 months. The first column indicates the child's age in months at the time of evaluation and the question type (alert or alarm). The second column shows the question the pediatrician asks the child's tutor to evaluate the child's state of language acquisition and the "System decision" column contains the system's answer in the event of a negative answer to the question (referral to specialist or bring forward visit).

Table 1. Shows KB questions for 1,2 and 3months

\begin{tabular}{|c|c|c|}
\hline Age-Milestone & Question to be answered by pediatrician & System decision \\
\hline 1 month Alarm & Reacts to a bell & Send to specialist to check hearing \\
\hline 1 month Alarm & Vocalizes without crying & Send to specialist to check hearing \\
\hline 2 months Alert & Emits "OOO/AAH" & Bring forward visit (three months) \\
\hline 2 months Alert & Screams to interact & Bring forward visit (three months) \\
\hline 3 months Alett & $\begin{array}{l}\text { Turns around or reacts (closing eyes) to a } \\
\text { clap }\end{array}$ & Bring forward visit (three months) \\
\hline 3 months Alert & Turns at the sound of mother's voice & Bring forward visit (three months) \\
\hline 3 months Alarm & Emits "OOO/AAH" & $\begin{array}{l}\text { Check if hearing problem ruled out } \\
\text { Refer to neuropediatrician }\end{array}$ \\
\hline 3 months Alarm & Laughs in response to stimulus & Refer to neuropediatrician \\
\hline
\end{tabular}


Protégé [9] was used in formalization of the knowledge model to create the KB and the inference engine needed to support decision making. The ontology of Protégé was built with a class hierarchy for the first 6 years, including a sub-hierarchy of classes for each month corresponding to the questions to be asked by the pediatrician.

\subsection{Web Tool for Accessing the DSS}

Objective verification of the proposal to refer to a specialist arising from possible detection of language development disorders, suggested by the KB, required development of a useful web tool to facilitate the work of experts and users of pediatrics in primary care. For this reason, the DSS was implemented, offering the final user a simple verification interface based on an internal connection of the $\mathrm{KB}$. The definition of the binary relationships established between ontology classes will sustain the system's reasoning process through axioms such as: If the child is 2 months old and we get a negative answer to the question "Emits OOO/AAH" or "Cries to interact", then "To anticipate the next visit in three months". Figure 4 shows in greater detail the questions the DSS proposes to the pediatrician for the language evaluation process in a two month-old child (Pegasus):

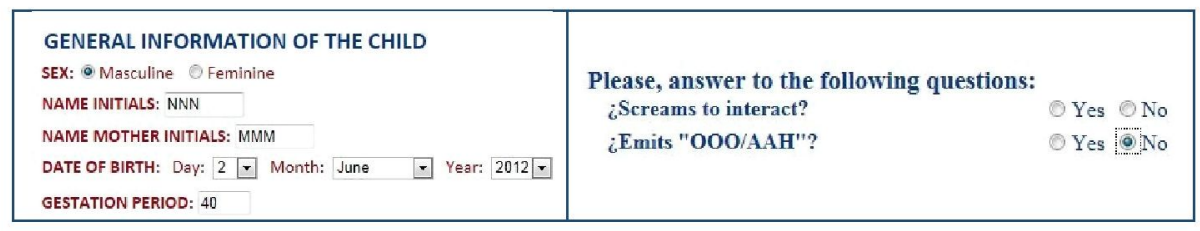

Fig. 4. Process of language evaluation

Figure 5 shows the result provided by the early detection tool based on a negative answer to the question "Emits OOO/AAH". At this point, the system proposes bringing forward the next visit and also suggests that the pediatrician enter an opinion on the system's decision or an alternative to the proposal of the system.

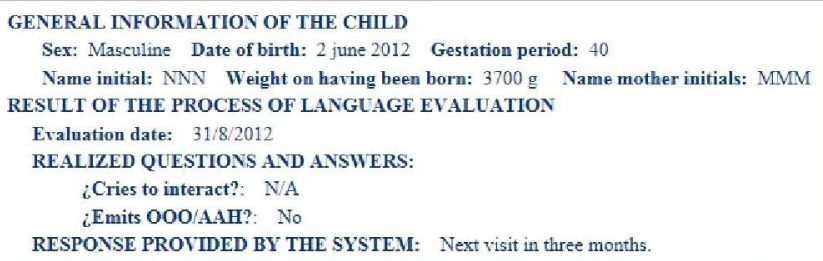

Fig. 5. Result of language evaluation process

\section{Conclusions}

The system proposes an innovative solution for the difficult task of detecting language disorders among children aged 0 to 6 in routine visits to pediatricians in primary care. Open web applications allow the easy utilization of the $\mathrm{KB}$ by pediatricians 
and specialist. The developed system does usable a DSS for early attention in primary care. The involvement of 4 experts in neuropediatrics, neonatology and language disorders was crucial for both defining the problem and for selecting 21 real, proven cases to refine the knowledge base with existing experience in primary care.

The opinion of the experts involved allows for a forthcoming start of the stage of validation, with a view to deployment and evaluation in routine clinical practice. The service evaluation is scheduled for autumn 2012 in cases considered of interest by the two pediatricians already involved in the final stage. The intelligent system is ripe for turn the withdrawal of information of the language acquisition state of the child by means of question - response, into an environmental intelligence based solutions.

Acknowledgements. Dr. José Arizcun, expert neonatologist in developmental disorders. Dra. $\mathrm{M}^{\mathrm{a}}$ Teresa Ferrando, neuropediatrician in the Quiron Hospital (Madrid). Dra. Beatriz Chiclana and Dr. Erwin Kirchschlager, pediatricians in the Jazmin Health Center. Dra. Teresa Díaz, pediatrician in the Quiron Hospital (Madrid). Ma Peñafiel and Paloma Tejeda of the Language Intervention Center (CIL) La Salle Campus Madrid, Universidad Autónoma de Madrid.

This article is part of research we're conducting in the Talisec+ project (a framework for knowledge-based management of accessible security guarantees for personal autonomy; TIN2010-20510-C04-01), supported by the Ministry of Education and Science of Spain through the National Plan for $\mathrm{R}+\mathrm{D}+\mathrm{I}$ (research, development, and innovation).

\section{References}

1. Council on Children With Disabilities, Section on Developmental Behavioral Pediatrics, Bright Futures Steering Committee and Medical Home Initiatives for Children With Special Needs Project Advisory Committee. Identifying infants and young children with developmental disorders in the medical home. Pediatrics (2006)

2. Arrabal Terán, M.C., Arizcun Pineda, J.: Alteraciones del desarrollo y discapacidad. Grado de pediatría en España. Genysi (2007)

3. Paul, R.: Language disorders from infancy through adolescence: Assessment and intervention. St. Louis. Mosby (2007)

4. Fejerman, N., Fernández Álvarez, E.: Neurología pediátrica. Capitulos, 51-54 (2007)

5. Narbona, J., Chevrie-Muller, C.: El lenguaje del niño. Desarrollo normal, evaluación y trastornos (2003)

6. Alonso Betanzos, A., Guijarro Berdiñas, B., Lozano Tello, A., Palma Méndez, J.T., Taboada Iglesias, M.J.: Ingeniería del conocimiento. Aspectos Metodológicos (2004)

7. Corcho, O., Fernández-López, M., Gómez-Pérez, A., López-Cima, A.: Building Legal Ontologies with METHONTOLOGY and WebODE. In: Benjamins, V.R., Casanovas, P., Breuker, J., Gangemi, A. (eds.) Law and the Semantic Web. LNCS (LNAI), vol. 3369, pp. 142-157. Springer, Heidelberg (2005)

8. Peñafiel Puerto, M.: Mejorando las habilidades en indicadores tempranos de los trastornos del lenguaje. FAPap, vol. 5 (2012)

9. Protégé (2000), Planning a Project http://protege.stanford.edu/doc/ users_guide/planning_a_project.html

10. Pellet OWL Reasoner for Java (2012),

http://clarkparsia.com/pellet/docs/ 